Pacific Journal of Mathematics

GLOBAL POSITIVE SOLUTIONS OF SEMILINEAR ELLIPTIC 


\title{
GLOBAL POSITIVE SOLUTIONS OF SEMILINEAR ELLIPTIC PROBLEMS
}

\author{
Ezzat S. Noussair and Charles A. Swanson
}

The existence of bounded positive solutions of semilinear elliptic boundary value problems of the type

$$
\begin{array}{ll}
L u=\lambda f(x, u), & x \in \Omega, \\
u(x)=0, & x \in \partial \Omega,
\end{array}
$$

will be proved in unbounded domains $\Omega \subset R^{n}, n \geq 2$, with boundary $\partial \Omega \in C^{2+\alpha}, 0<\alpha<1$, where $\lambda$ is a positive constant and

$$
L u=-\sum_{i, j=1}^{n} D_{l}\left[a_{\imath \jmath}(x) D_{j} u\right]+m(x) u, \quad x \in \Omega,
$$

$D_{l}=\partial / \partial x_{i}, i=1, \ldots, n$. The existence of a bounded positive solution of (1.1) in the entire space $R^{n}$ is proved also by the same procedure. The regularity and additional hypotheses H1-H5 to be imposed on $L$ and $f$ are stated in \$2. In particular, the assumption $f(x, 0)=0$ for all $x \in \Omega$ implies that the boundary value problem (1.1), (1.2) always has the trivial solution.

1. Introduction. Generally a positive solution of (1.1), (1.2) in $\Omega$ exists only if $\lambda$ is sufficiently large, as might be expected from known results for bounded domains, see e.g. Rabinowitz [23]. In fact, under the extra hypotheses $\mathrm{H} 8$ and $\mathrm{H} 9$, we prove the Uniqueness Theorem 5.1: There exists a positive interval $\left(0, \lambda_{*}\right]$ such that (1.1), (1.2) has no nontrivial solution $u(x, \lambda)$ for any $\lambda$ in this interval. However, under different conditions $\mathrm{H} 6$ and $\mathrm{H} 7$, Theorems 4.4 and 4.6 yield bounded positive solutions of, respectively, the boundary value problem (1.1), (1.2) and the differential equation (1.1) in all of $R^{n}$ for arbitrary positive $\lambda$.

The physically important case $[9,25]$

$$
-\Delta u+m(x) u=p(x) u^{\gamma}-q(x) u^{\beta}, \quad x \in \Omega,
$$

is included, where $1<\gamma<\beta$ and $p, q, m$ are nonnegative functions in $\Omega$. Solutions $u(x)$ of (1.4) provide stationary states $e^{i \omega t} u(x)$ of the corresponding wave equation, often called the Klein-Gordon equation. In the case of constants $p, q, m$, the existence of positive solutions of (1.4) in the 
entire space $R^{n}$ which decay exponentially at $\infty$ (if $m>0$ ) has been proved by Berestycki and Lions [7], Berestycki, Lions, and Peletier [8], Berger [9], and Strauss [25]. These results of course are not concerned with boundary conditions on $\partial \Omega$. Our method establishes, in particular, the existence of positive solutions of the Dirichlet problem for (1.4) in unbounded domains $\Omega$ without the requirement of any symmetry conditions on either $\Omega$ or the coefficients. The earlier methods cited, depending critically on the radial symmetry of the coefficients, could not be extended to solve the present problems.

However, we are unable to prove in general that the solution has limit zero at $\infty$. This is proved in $\S 5$ only in cases for which the nonlinear terms in (1.4) have limit zero at $\infty$.

Existence theorems for boundary value problems on bounded domains are contained in papers by Ako [1], Amann [2], Amann and Crandall [3], Bandle [4], Keller [16], Nagumo [18], Rabinowitz [23], and Schmitt [24]. Extensive bibliographies appear in [23] and [24]. For unbounded domains, the existence theorems of Benci and Fortunato [5, 6], Berger and Schechter [10], Bose [11], Edmunds and Evans [13], Edmunds and Webb [14], Noussair [19] and Ogata [21] either apply only to special cases of our problem, or do not guarantee nontrivial solutions, or both. The complex theory of weighted Sobolev spaces, as developed and exploited in $[\mathbf{5 , 6}, \mathbf{1 0}$, $13,14]$, requires restrictions on the "size" of the domain $\Omega$ at $\infty$ and does not aim at positivity or asymptotic behavior of the solutions.

Section 2 describes the notation and principal hypotheses. In $\S 3$ some results of Rabinowitz [23] for bounded domains are adapted to our structure. The main Theorem 4.3 establishes the existence of a bounded positive solution of (1.1), (1.2) in $\Omega$ for all sufficiently large $\lambda$ if $\mathrm{H} 1-\mathrm{H} 5$ hold. The modifications in Theorem 4.5 and 4.6 yield bounded positive solutions of (1.1) in $R^{n}$. Section 5 concerns the existence of positive solutions $u(x, \lambda)$ of (1.1), (1.2) which have limit 0 as $|x| \rightarrow \infty$. This is proved in two cases: (i) $m(x)$ is uniformly positive and $f(x, t) \rightarrow 0$ as $|x| \rightarrow \infty$; and (ii) in dimensions $n \geq 3, f(x, t)$ satisfies a Dini condition of the Meyers and Serrin type [17]. Section 6 contains modifications for $m(x) \leq 0$.

The case of nonnegative functions $f(x, u)$ for all $x, u$ is not being considered here. We solve (1.1), (1.2) in this case elsewhere by completely different methods.

2. Preliminaries. Let $|x|$ denote the Euclidean norm of a point $x=\left(x_{1}, \ldots, x_{n}\right)$ in real Euclidean $n$-space $R^{n}, n \geq 2$. The notation below 
will be used throughout:

$$
\begin{array}{ll}
S_{t}=\left\{x \in R^{n}:|x|=t\right\}, & t>0, \\
G_{t}=\left\{x \in R^{n}:|x|>t\right\}, & t>0, \\
G_{s, t}=\left\{x \in R^{n}: s<|x|<t\right\}, & 0<s<t, \\
\Omega_{t}=\{x \in \Omega:|x|<t\}, & t>0 .
\end{array}
$$

An exterior domain $\Omega \subset R^{n}$ has the property that $G_{\delta} \subset \Omega$ for some $\delta>0$, fixed in the sequel.

For a bounded domain $M \subset \Omega, C^{m+\alpha}(\bar{M})$ denotes the usual Hölder space, with norm $\|\cdot\|_{m+\alpha, \bar{M}}, 0<\alpha<1, m=0,1,2, \ldots$ The abbreviation $C_{\mathrm{loc}}^{m+\alpha}(\Omega)$ is used for the set of all $u \in C^{m+\alpha}(\bar{M})$ for every bounded subdomain $M$ of $\Omega$. The space $\stackrel{\circ}{2}_{2}^{1}(M)$ is defined to be the completion of $C_{0}^{\infty}(M)$ in the norm

$$
\|u\|=\left[\int_{M} \sum_{|i| \leq 1}\left|D^{i} u(x)\right|^{2} d x\right]^{1 / 2}
$$

where multi-index notation has been used.

The conditions below will be imposed on the functions $a_{l j}, m$, and $f$ in (1.1), (1.3):

H1. Each $a_{i j} \in C_{\mathrm{loc}}^{1+\alpha}(\Omega), m \in C_{\mathrm{loc}}^{\alpha}(\Omega), m(x) \geq 0$ in $\Omega$, and $\left(a_{i j}(x)\right)$ is symmetric and uniformly positive definite in every bounded subdomain of $\Omega$, where $\alpha \in(0,1)$ is fixed throughout.

H2. $f$ is locally Lipschitz continuous on $(\Omega \cup \partial \Omega) \times R^{+}$, where $R^{+}=$ $[0, \infty)$, and $f(x, 0)=0$ for all $x \in \Omega$.

H3. There exists a positive number $T$ such that $f(x, t)<0$ for all $t>T$ and for all $x \in \Omega$.

H4. There exist $x_{0} \in \Omega$ and $T_{0} \in[0, T)$ such that $F\left(x_{0}, T_{0}\right)>0$, where

$$
F(x, t)=\int_{0}^{t} f(x, \tau) d \tau, \quad 0 \leq t<\infty
$$

H5. For every bounded domain $M \subset \Omega$ and for every $t_{0}>0$, there corresponds a positive constant $K=K\left(M, t_{0}\right)$ such that $\lambda f(x, t)+K t$ is a nondecreasing function of $t$ on $0 \leq t \leq t_{0}$ for each fixed $x \in \bar{M}$. 
H5 is satisfied, for example, if $f(x, t)$ is continuously differentiable with respect to $t$ at every $(x, t) \in \Omega \times R^{+}$. In particular $\mathrm{H} 2$ implies that $f \in C_{\mathrm{loc}}^{\alpha}\left(\Omega \times R^{+}\right)$.

These hypotheses are all satisfied in the case that (1.1) is specialized to equation (1.4) under the following conditions: (i) $1<\gamma<\beta$; (ii) $p, q$, $m$ are locally Lipschitz continuous, nonnegative, and bounded; and (iii) $p / q$ is bounded and bounded away from zero in $\Omega$.

3. Construction of subsolutions. Let $T$ be as in $\mathrm{H} 3$ and let $f_{T}$ : $\Omega \times R \rightarrow R$ be defined by

$$
f_{T}(x, t)= \begin{cases}0 & \text { if } t<0 \\ f(x, t) & \text { if } 0 \leq t \leq T \\ f(x, T) & \text { if } t>T\end{cases}
$$

Also define $F_{T}$ by

$$
F_{T}(x, t)=\int_{0}^{t} f_{T}(x, \tau) d \tau .
$$

Then, by (2.1) and (3.2), $F_{T}(x, t)=F(x, t)$ for $0 \leq t \leq T, x \in \Omega$. Associated with (1.1), consider the following boundary value problem on bounded domains $\Omega_{R}$ :

$$
\begin{cases}L u=\lambda f(x, u), & x \in \Omega_{R}, \\ u(x)=0, & x \in \partial \Omega_{R} .\end{cases}
$$

Let $I_{R}(\phi, \lambda)$ be the functional on $\stackrel{\circ}{W}_{2}^{1}\left(\Omega_{R}\right)$ defined by

$$
I_{R}(\phi, \lambda)=I_{1, R}(\phi)-\lambda I_{2, R}(\phi),
$$

where

$$
\begin{aligned}
& I_{1, R}(\phi)=\frac{1}{2} \int_{\Omega_{R}}\left[\sum_{i, j=1}^{n} a_{i j}(x) D_{i} \phi D_{j} \phi+m(x) \phi^{2}(x)\right] d x \\
& I_{2, R}(\phi)=\int_{\Omega_{R}} F_{T}(x, \phi(x)) d x .
\end{aligned}
$$

THEOREM 3.1 (Rabinowitz). If $\mathrm{H} 1-\mathrm{H} 4$ hold, there exist positive constants $R$ and $\lambda^{*}=\lambda^{*}(R)$ such that the boundary value problem (3.3) has a positive solution $u_{R}=u_{R}(x, \lambda)$ in $\Omega_{R}$ for all $\lambda \geq \lambda^{*}$. Furthermore, the functional $I_{R}(\phi, \lambda)$ on $\stackrel{\circ}{W}_{2}^{1}\left(\Omega_{R}\right)$ attains its minimum at $\phi=u_{R}(\cdot, \lambda)$ for $\lambda \geq \lambda^{*}$. 
H4 implies that $R$ can be chosen large enough (and fixed in the sequel) so that there exists a point $x_{0} \in \Omega_{R}$ with $F\left(x_{0}, T_{0}\right)>0$. Then Theorem 3.1 follows from a theorem of Rabinowitz [23, p. 177] applied to the bounded domain $\Omega_{R}$.

Theorem 3.1 is strengthened to the result below under the following additional hypotheses:

H6. Each $a_{i j}(x)$ and $f(x, t)$ are bounded in $\Omega$ for each fixed $t>0$, and $m(x)=0$ identically.

H7. There exists a positive constant $C$ such that

$$
\limsup _{R \rightarrow \infty} R^{1-n} \int_{\Omega_{R}} F(x, C) d x=+\infty,
$$

where $F(x, t)$ is defined by (2.1).

THEOREM 3.2. If $\mathrm{H} 1-\mathrm{H} 3, \mathrm{H} 6$, and $\mathrm{H} 7$ hold, there exists $R>0$ such that (3.3) has a nontrivial nonnegative solution $u_{R}(x, \lambda)$ in $\Omega_{R}$ for all $\lambda>0$.

Proof. $\mathrm{H} 7$ implies that $\mathrm{H} 4$ holds for some $x_{0}$ in a domain $\Omega_{R}$ for some $R>0$. The proof given by Rabinowitz [23, pp. 176-177] shows on the basis of $\mathrm{H} 1-\mathrm{H} 4$ in $\Omega_{R}$ that $I_{R}(\phi, \lambda)$ attains its minimum on $\stackrel{\circ}{W}_{2}^{1}\left(\Omega_{R}\right)$ at a point $\phi=u_{R}(\cdot, \lambda)$ which is a nonnegative classical solution of (3.3) in $\Omega_{R}$. To show that $u_{R}$ is nontrivial, consider the piecewise $C^{1}$ function on $\bar{\Omega}_{R}$ defined by

$$
w_{R}(x)= \begin{cases}0 & \text { if } x \in \bar{\Omega}_{a}, \\ C(|x|-a) & \text { if } a \leq|x| \leq a+1, \\ C & \text { if } a+1 \leq|x| \leq R-1, \\ C(R-|x|) & \text { if } R-1 \leq|x| \leq R,\end{cases}
$$

where $a \geq 0$ is chosen so that $G_{a} \subset \Omega$, and $R>a+2$ without loss of generality. Since $w_{R}=0$ on $\partial \Omega_{R}, w_{R} \in \stackrel{\circ}{W}_{2}^{1}\left(\Omega_{R}\right)$. Each $a_{l j}(x)$ and $F_{T}(x, t)$ are bounded in $\Omega$ for each fixed $t>0$ by H6, (3.1), and (3.2). Then one sees easily from (3.5) and (3.6) that there exist positive constants $K_{1}$ and $K_{2}$ such that

$$
\begin{aligned}
& I_{1, R}\left(w_{R}\right) \leq K_{1} R^{n-1} \\
& I_{2, R}\left(w_{R}\right) \geq-K_{2} R^{n-1}+\int_{G_{a+1, R-1}} F_{T}(x, C) d x,
\end{aligned}
$$


for $R>a+2$. Since $C<T$ by H3 and H7, $F_{T}(x, C)=F(x, C)$. Then $\mathrm{H} 7$ and (3.4) show that $I_{R}\left(w_{R}, \lambda\right)<0$ for some sufficiently large $R$. However, $I_{R}(0, \lambda)=0$ from $\mathrm{H} 2,(3.2),(3.4)-(3.6)$ and hence $u_{R}$ is nontrivial from the minimum property of $u_{R}$.

REMARK. The boundedness hypothesis $\mathrm{H} 6$ can be relaxed to allow unbounded functions $a_{i j}(x)$ and $f(x, \cdot)$ provided $\mathrm{H} 7$ is modified. This can be accomplished by a different choice of $w_{R}(x)$ in the proof of Theorem 3.2.

4. Existence of positive solutions. A solution of (1.1), (1.2) in an unbounded domain $\Omega \subset R^{n}$ is understood to be a function $u=u(\cdot, \lambda) \in$ $C_{\text {loc }}^{2+\alpha}(\Omega)$ such that $u$ satisfies (1.1), (1.2) identically.

Let $u_{R}(x, \lambda)$ be a nontrivial nonnegative solution of the boundary value problem (3.3) in a bounded domain $\Omega_{R}$. If $\mathrm{H} 1-\mathrm{H} 4$ are satisfied, Theorem 3.1 shows that $u_{R}(x, \lambda)$ exists for some $R>0$ (fixed) and for all $\lambda \geq \lambda^{*}>0$. If $\mathrm{H} 1-\mathrm{H} 3, \mathrm{H} 6$, and $\mathrm{H} 7$ hold, Theorem 3.2 shows that $u_{R}(x, \lambda)$ exists for all $\lambda>0$. Let $w_{0}(x, \lambda)$ denote the extension of $u_{R}(x, \lambda)$ to all of $R^{n}$ defined by

$$
w_{0}(x, \lambda)= \begin{cases}u_{R}(x, \lambda) & \text { if } x \in \bar{\Omega}_{R} \\ 0 & \text { otherwise }\end{cases}
$$

The Lemma below concerns functions $v \in C_{\mathrm{loc}}^{2+\alpha}(\Omega)$ (to be found later) which satisfy

$$
L v \geq \lambda f(x, v) \quad \text { in } \Omega, \quad v \geq 0 \quad \text { on } \partial \Omega .
$$

LEMMA 4.1. Under hypotheses $\mathrm{H} 1, \mathrm{H} 2$, and $\mathrm{H} 5$, suppose there exist non-trivial functions $u_{R} \in C^{2+\alpha}\left(\bar{\Omega}_{R}\right)$ and $v \in C_{\operatorname{loc}}^{2+\alpha}(\Omega)$ satisfying (3.3) and (4.2), respectively, such that $0 \leq u_{R}(x, \lambda) \leq v(x, \lambda)$ for all $x \in \bar{\Omega}_{R}, R>0$, $\lambda>0$. Then there exists a sequence of functions $w_{j} \in C^{2+\alpha}\left(\bar{\Omega}_{R+j}\right)$ satisfying

$$
L w_{j} \leq \lambda f\left(x, w_{j}\right) \quad \text { in } \Omega_{R+j}, \quad w_{J}=0 \quad \text { on } \partial \Omega_{R+j}
$$

such that $w_{0}(x, \lambda) \leq w_{j}(x, \lambda) \leq v(x, \lambda)$ throughout $\bar{\Omega}_{R+j}$, where $w_{0}(x, \lambda)$ is defined by (4.1).

Proof. Define

$$
T_{j}=\sup _{x \in \Omega_{R+j}} v(x), \quad j=1,2, \ldots
$$


By $\mathrm{H} 5$, we can choose a positive constant $K_{j}$, depending on $v$, such that $\lambda f(x, t)+K_{j} t$ is a nondecreasing function of $t$ on $0 \leq t \leq T_{j}$ for each fixed $x \in \bar{\Omega}_{R+\jmath}$. Define $w_{j}=w_{j}(x, \lambda)$ to be the unique solution of the linear boundary value problem

$$
\left\{\begin{array}{ll}
\left(L+K_{j}\right) w_{j}=\lambda f\left(x, w_{0}(x)\right)+K_{J} w_{0}(x) & \text { in } \Omega_{R+\jmath} \\
w_{J}(x)=0 & \text { on } \partial \Omega \cup S_{R+\jmath}
\end{array} .\right.
$$

We now verify that $w_{j}$ has all the properties stated in Lemma 4.1. Since $\lambda$ is fixed, we delete the $\lambda$ dependence from the notation $w_{J}(x, \lambda)$, etc.

First let $F_{j}(x)=\lambda f\left(x, w_{0}(x)\right)+K_{j} w_{0}(x), x \in \bar{\Omega}_{R+j}$. Then $F_{j} \in$ $C^{\alpha}\left(\bar{\Omega}_{R+j}\right)$ by $\mathrm{H} 2$, and the standard Schauder estimate for the solution of (4.3) $\left[12\right.$, p. 335] shows that $w_{j} \in C^{2+\alpha}\left(\bar{\Omega}_{R+j}\right), j=1,2, \ldots$

It follows from (4.2) and (4.3) that

$$
\left(L+K_{j}\right)\left(v-w_{J}\right) \geq\left[\lambda f(x, v)+K_{j} v\right]-\left[\lambda f\left(x, w_{0}\right)+K_{j} w_{0}\right]
$$

in $\Omega_{R+j}$. Since $v \geq u_{R}$ in $\bar{\Omega}_{R}$ by hypothesis, so also $v \geq w_{0}$ in $\Omega_{R+\jmath}$, $\left(L+K_{J}\right)\left(v-w_{j}\right) \geq 0$ in $\Omega_{R+j}$. Furthermore, $v-w_{J}=v \geq 0$ on $\partial \Omega \cup$ $S_{R+j}$ and therefore $v-w_{j} \geq 0$ throughout $\bar{\Omega}_{R+j}$ by the maximum principle [22].

Similarly, $\left(L+K_{j}\right) w_{j} \geq 0$ in $\Omega_{R+j}$ and hence $w_{J} \geq 0$ throughout $\Omega_{R+j}$. From (3.3) and (4.3) we obtain

$$
\begin{cases}\left(L+K_{J}\right)\left(w_{J}-u_{R}\right)=0 & \text { in } \Omega_{R}, \\ w_{j}-u_{R}=0 & \text { on } \partial \Omega, \\ w_{J}-u_{R}=w_{J} \geq 0 & \text { on } S_{R},\end{cases}
$$

from which $w_{j} \geq u_{R}$ throughout $\bar{\Omega}_{R}$ by the maximum principle, and hence $w_{j} \geq w_{0}$ throughout $\Omega \cup \partial \Omega$ by (4.1).

Finally, from (4.3),

$$
L w_{j}-\lambda f\left(x, w_{j}\right)=\left[\lambda f\left(x, w_{0}\right)+K_{J} w_{0}\right]-\left[\lambda f\left(x, w_{J}\right)+K_{J} w_{J}\right] \leq 0
$$

in $\Omega_{R+j}, j=1,2, \ldots$

LEMMA 4.2. Under the hypotheses of Lemma 4.1, there exists a sequence of functions $u_{j}$ in $\Omega \cup \partial \Omega$ with the following properties:
(A) $u_{J} \in C^{2+\alpha}\left(\bar{\Omega}_{R+j}\right)$;
(B) $u_{j}(x, \lambda)=0$ if $x \in \partial \Omega \cup S_{R+j} \cup G_{R+j}$;
(C) $L u_{j}=\lambda f\left(x, u_{j}(x, \lambda)\right)$ if $x \in \Omega_{R+j}$;
(D) $w_{0}(x, \lambda) \leq w_{j}(x, \lambda) \leq u_{j}(x, \lambda) \leq v(x, \lambda)$ in $\bar{\Omega}_{R+j}$;
(E) $u_{j+1}(x, \lambda) \geq u_{j}(x, \lambda)$ in $\Omega \cup \partial \Omega, j=1,2, \ldots$ 
Proof. By Lemma 4.1, $w_{j}$ is a subsolution and $v$ is a supersolution of the boundary value problem

$$
\begin{cases}L u=\lambda f(x, u), & x \in \Omega_{R+j}, \\ u(x)=0, & x \in \partial \Omega_{R+j},\end{cases}
$$

with $w_{j}(x, \lambda) \leq v(x, \lambda)$ throughout $\bar{\Omega}_{R+j}$. Then a theorem of Amann [2, p. 283] shows that (4.4) has a minimal solution $u=U_{j} \in C^{2+\alpha}\left(\bar{\Omega}_{R+j}\right)$ satisfying $w_{0} \leq w_{J} \leq U_{j} \leq v$ throughout $\bar{\Omega}_{R+j}$, i.e. $W_{j} \geq U_{j}$ on $\bar{\Omega}_{R+j}$ for any solution $W_{j}$ of (4.4) satisfying $w_{j} \leq W_{j} \leq v$ on $\bar{\Omega}_{R+\jmath}$. The extension $u_{j}(x, \lambda)$ of $U_{j}(x, \lambda)$ to $\Omega \cup \partial \Omega$ defined to be 0 for $|x|>R+j$ then has properties (A)-(D) of the Lemma.

To prove (E), notice from (A)-(D) that $u_{j+1}$ is a supersolution of (4.4) in $\Omega_{R+j}$. By Amann's Theorem [2] again, there exists a solution $W_{J}$ of (4.4) satisfying $w_{0} \leq w_{j} \leq W_{j} \leq u_{j+1}$ in $\Omega_{R+j}$. The minimality of $U_{j}$ then implies that $W_{j} \geq U_{j}$, and hence $u_{j+1} \geq u_{j}$ in $\bar{\Omega}_{R+j}$. Since the same inequality holds if $|x|>R+j$ by (B), (D), property (E) has been proved.

In the first main theorem we specialize Lemma 4.2 to the case of the constant function $v(x, \lambda)=T$, which evidently satisfies (4.2) if H3 holds.

THEOREM 4.3. If $\mathrm{H} 1-\mathrm{H} 5$ are satisfied, there exists $\lambda^{*}>0$ such that the boundary value problem (1.1), (1.2) has a bounded positive solution $u(x, \lambda)$ in $\Omega$ satisfying $w_{0}(x, \lambda) \leq u(x, \lambda) \leq T$ for all $x \in \Omega \cup \partial \Omega$ and for all $\lambda \geq \lambda^{*}$.

Proof. Since H1-H4 hold, Theorem 3.1 guarantees a positive solution $u_{R}(x, \lambda)$ of (3.3) in $\Omega_{R}$ for all $\lambda \geq \lambda^{*}>0$, as required for Lemma 4.2. We need to check first that the constant solution $v(x, \lambda)=T$ of (4.2) satisfies $u_{R}(x, \lambda) \leq T$ for all $x \in \bar{\Omega}_{R}$. Suppose to the contrary that $u_{R}\left(x_{0}, \lambda\right)>T$ is a (necessarily positive) maximum of $u_{R}(x, \lambda)$ in $\Omega_{R}$. Then, in appropriate coordinates, $0 \leq L u_{R}\left(x_{0}, \lambda\right)=\lambda f\left(x_{0}, u_{R}\left(x_{0}, \lambda\right)\right)$, contradicting H3. Accordingly it follows from (4.1) that $w_{0}(x, \lambda) \leq T$ for all $x \in \Omega$.

Therefore the sequence $\left\{u_{j}(x, \lambda)\right\}$ in Lemma 4.2 exists. The next (crucial) step is to prove, for every positive integer $i$, that there exists a positive constant $K(i)$, independent of $j \geq i$, such that

$$
\left\|u_{j}\right\|_{2+\alpha, \bar{\Omega}_{R+1}} \leq K(i) \text { for all } j \geq i .
$$

This can be accomplished by $L^{p}$-estimates, Sobolev embedding, and Schauder estimates as in [20, Lemma 3.2]. Then the compactness of the injection $C^{2+\alpha}\left(\bar{\Omega}_{R+i}\right) \rightarrow C^{2}\left(\bar{\Omega}_{R+i}\right)$ enables us to define a subsequence 
$\left\{u_{j}^{i}\right\}$ of $\left\{u_{j}^{i-1}\right\}$ inductively which converges in the $C^{2}\left(\bar{\Omega}_{R+l}\right)$ norm to a function $u^{i} \in C^{2}\left(\bar{\Omega}_{R+i}\right), i=2,3, \ldots$, where $u_{j}^{1}=u_{j}$. The proof given in $\left[20\right.$, p. 126] shows that the diagonal sequence $\left\{u_{J}^{J}\right\}$ converges to a solution $u$ of (1.1), (1.2). By property (D) of Lemma 4.2, the solution satisfies $w_{0}(x, \lambda) \leq u(x, \lambda) \leq T$ in $\Omega$, and so is bounded.

The pointwise limit of $\left\{u_{j}(x, \lambda)\right\}$ exists by the monotone property (E), and the limit can only be the function $u(x, \lambda)$ constructed above. To prove that $u(x, \lambda)>0$ for all $x \in \Omega$, let $M$ be any bounded domain with $\bar{M} \subset \Omega$ and choose an integer $i$ such that $\bar{M} \subset \Omega_{R+i}$. By Lemma 4.1, $w_{l}$ is nontrivial and satisfies

$$
\left(L+K_{l}\right) w_{i} \geq 0 \quad \text { in } \Omega_{R+l}, \quad w_{l}=0 \quad \text { on } \partial \Omega_{R+i}
$$

for $K_{i}>0$. Then the strong maximum principle [22] shows that $w_{i}>0$ throughout $\Omega_{R+\imath}$. By properties (D) and (E) of Lemma 4.2, $u_{\jmath} \geq u_{\imath} \geq w_{i}$ throughout $\Omega_{R+i}$ for all $j \geq i$, and therefore $u(x, \lambda)=\lim _{j \rightarrow \infty} u_{j}(x, \lambda)>0$ on $\bar{M}$. Since $M$ is arbitrary, $u(x, \lambda)$ is a positive solution of $(1.1),(1.2)$ in $\Omega$ for all $\lambda \geq \lambda^{*}$.

The following analogue of Theorem 4.3 is proved in the same way by using Theorem 3.2 instead of Theorem 3.1.

THEOREM 4.4. Suppose that $\mathrm{H} 1-\mathrm{H} 3$ and $\mathrm{H} 5-\mathrm{H} 7$ are satisfied. Then the boundary value problem (1.1), (1.2) has a bounded positive solution $u(x, \lambda)$ in $\Omega$ satisfying $w_{0}(x, \lambda) \leq u(x, \lambda) \leq T$ for all $x \in \Omega \cup \partial \Omega$ and for all $\lambda>0$.

The existence of a bounded positive solution of (1.1) in the entire space $R^{n}$ is proved by exactly the same method. An analogue of Lemma 4.2 , with $\Omega$ replaced by $R^{n}$ and $\partial \Omega$ deleted, follows from Theorem 3.1 or Theorem 3.2 without essential change. The hypotheses $\mathrm{H}^{\prime} 1-\mathrm{H}^{\prime} 7$ are formally the same as $\mathrm{H} 1-\mathrm{H} 7$ with $R^{n}$ replacing $\Omega$ and $\partial \Omega$ deleted.

THEOREM 4.5. If $\mathrm{H}^{\prime} 1-\mathrm{H}^{\prime} 5$ hold, there exists $\lambda^{*}>0$ such that (1.1) has $a$ bounded positive solution $u(\cdot, \lambda) \in C_{\mathrm{loc}}^{2+\alpha}\left(R^{n}\right)$ satisfying $w_{0}(x, \lambda) \leq$ $u(x, \lambda) \leq T$ for all $x \in R^{n}$ and for all $\lambda \geq \lambda^{*}$.

THEOREM 4.6. If $\mathrm{H}^{\prime} 1-\mathrm{H}^{\prime} 3$ and $\mathrm{H}^{\prime} 5-\mathrm{H}^{\prime} 7$ are satisfied, then (1.1) has a bounded positive solution $u(x, \lambda)$ satisfying $w_{0}(x, \lambda) \leq u(x, \lambda) \leq T$ for all $x \in R^{n}$ and for all $\lambda>0$.

5. Behavior of solutions as $|x| \rightarrow \infty$. In this section we consider the case that (1.1) is the Schrödinger equation. Then the boundary value 
problem $(1.1),(1.2)$ reduces to

$$
\begin{array}{ll}
-\Delta u+m(x) u=\lambda f(x, u), & x \in \Omega, \\
u(x)=0, & x \in \partial \Omega .
\end{array}
$$

We prove in particular, under hypotheses selected from the list below, that (5.1), (5.2) has an exponentially decaying positive solution $u(x, \lambda)$ in $\Omega$ as $|x| \rightarrow \infty$, provided $\lambda$ is sufficiently large.

H8. There exists $m_{0}>0$ such that $m(x) \geq m_{0}>0$ for all $x \in \Omega$.

H9. $f(x, t) / t$ is bounded in $\Omega \times(0, T]$, and $\lim _{t \rightarrow 0+}(f(x, t) / t)=0$ for all $x \in \Omega$.

H10. $\lim _{|x| \rightarrow \infty} \sup _{0<t \leq T}(f(x, t) / t)=0$.

H11. There exists $r_{0}>0$ such that

$$
\sup _{|x|=r} \phi(x) \leq \frac{\delta(r)}{r^{2}} \quad \text { for all } r \geq r_{0},
$$

where

$$
\phi(x)=\sup _{0 \leq t \leq T} f(x, t)
$$

and $\delta(r)$ denotes a Dini function, i.e. the improper integral $\int^{\infty} r^{-1} \delta(r) d r$ exists and is finite.

UniQueness THEOREM 5.1. If $\mathrm{H} 1-\mathrm{H} 4, \mathrm{H} 8$, and $\mathrm{H} 9$ hold, then there exists $\lambda_{*}>0$ such that the only nonnegative bounded solution $u(x, \lambda)$ of (5.1), (5.2) is identically zero in $\Omega$ for $0<\lambda \leq \lambda_{*}$.

Proof. Define $h(x, t)=f(x, t) / t$ if $t>0, h(x, 0)=0$ for all $x \in \Omega$, and

$$
\mu_{*}=\sup _{\substack{x \in \Omega \\ 0 \leq t \leq T}} h(x, t), \quad \lambda_{*}=\frac{m_{0}}{2 \mu_{*}} .
$$

Thus $\mu_{*}$ is positive and finite by $\mathrm{H} 4$ and $\mathrm{H} 9$, so $\lambda_{*}>0$. For any nonnegative bounded solution $u(x, \lambda)$ of $(5.1),(5.2)$, define

$$
M(x, \lambda)=m(x)-\lambda h(x, u(x, \lambda)), \quad x \in \Omega .
$$


If $0<\lambda \leq \lambda_{*}$ and $0 \leq u(x, \lambda) \leq T$ it follows that

$$
\lambda h(x, u(x, \lambda)) \leq \lambda_{*} \mu_{*}=m_{0} / 2, \quad x \in \Omega,
$$

and hence by $\mathrm{H} 8$ that

$$
M(x, \lambda) \geq m(x)-m_{0} / 2 \geq m_{0} / 2>0, \quad x \in \Omega .
$$

However by H3, $h(x, u(x, \lambda))<0$ for all $x \in \Omega$ such that $u(x, \lambda)>T$, and for all $\lambda>0$, and so $M(x, \lambda)>m(x) \geq m_{0}$ at all such points. Therefore (5.4) is satisfied for all $x \in \Omega$ and for all $\lambda$ in $0<\lambda \leq \lambda_{*}$.

By (5.1) and (5.3), $u(x, \lambda)$ is a solution of the linear differential equation

$$
-\Delta u+M(x, \lambda) u=0, \quad x \in \Omega .
$$

Since $u(x, \lambda)$ is bounded, a theorem of Kato [15, p. 415] shows in view of (5.4) that there exists a positive constant $C(\lambda)$ such that

$$
0 \leq u(x, \lambda) \leq C(\lambda) \exp \left[-\sqrt{m_{0} / 2}|x|\right], \quad x \in \Omega,
$$

for $0<\lambda \leq \lambda_{*}$. Because of the boundary condition (5.2) on $\partial \Omega, u(x, \lambda)$ must have a local positive maximum at some point $x_{0} \in \Omega$ unless $u(x, \lambda)$ is identically zero in $\Omega$. The conclusion of Theorem 5.1 then follows from the maximum principle for (5.5).

TheOREM 5.2. Suppose $\mathrm{H} 1-\mathrm{H} 5, \mathrm{H} 8$, and $\mathrm{H} 10$ are satisfied. Then there exists $\lambda^{*}>0$ such that, for all $\lambda \geq \lambda^{*}$, the boundary value problem (5.1), (5.2) has a positive solution $u(x, \lambda)$ in $\Omega$ satisfying $u(x, \lambda) \leq$ $C(\lambda) \exp \left[-\sqrt{m_{0} / 2}|x|\right]$ for some constant $C(\lambda)>0$.

Proof. Let $u(x, \lambda)$ be the solution of (5.1), (5.2) constructed in Theorem 4.3: $0<u(x, \lambda) \leq T$ for all $x \in \Omega, \lambda \geq \lambda^{*}, \lambda^{*}$ as in Theorem 4.3. Define

$$
H(x)=\sup _{0<t \leq T} h(x, t), \quad h(x, t)=f(x, t) / t .
$$

By H10 there exists $\rho(\lambda)>0$ such that $\lambda H(x) \leq m_{0} / 2$ for all $x \in \Omega$ with $|x| \geq \rho(\lambda)$. Since $0<u(x, \lambda) \leq T$ for all $x \in \Omega$, it follows that

$$
\lambda h(x, u(x, \lambda)) \leq m_{0} / 2 \text { for }|x| \geq \rho(\lambda), \lambda \geq \lambda^{*} .
$$

Then $\mathrm{H} 8$ and (5.3) imply that

$$
M(x, \lambda) \geq m_{0} / 2 \text { for }|x| \geq \rho(\lambda), \lambda \geq \lambda^{*} .
$$

Since $u(x, \lambda)$ is a solution of the linear equation (5.5) with (5.6) holding in $G_{\rho(\lambda)}$, Kato's Theorem [15, p. 415] implies that there exists a constant 
$C_{0}(\lambda)>0$ for $\lambda \geq \lambda^{*}$ such that

$$
0<u(x, \lambda) \leq C_{0}(\lambda) \exp \left[-\sqrt{m_{0} / 2}|x|\right], \quad|x| \geq \rho(\lambda) .
$$

The estimate in Theorem 5.2 then follows, where $C(\lambda)=\max \left[C_{0}(\lambda), T\right]$.

A version of the Dirichlet problem for unbounded domains is

$$
\begin{cases}-\Delta u+m(x) u=\lambda f(x, u), & x \in \Omega, \\ u(x)=0, & x \in \partial \Omega, \\ u(x) \rightarrow 0 & \text { as }|x| \rightarrow \infty .\end{cases}
$$

Under the hypotheses of Theorem 5.2, a positive solution $u(x, \lambda)$ of $(5.7)$ in $\Omega$ exists for all $\lambda \geq \lambda^{*}$.

We now obtain an analogue of Theorem 5.2 without the uniform positivity hypothesis $\mathrm{H} 8$ on the coefficient $m(x)$ in (5.1). In particular, $m(x)$ is allowed to be identically zero.

THEOREM 5.3. If $\mathrm{H} 1-\mathrm{H} 5$ and $\mathrm{H} 11$ hold, and $n \geq 3$, then there exists $\lambda^{*}>0$ such that (5.1), (5.2) has a positive solution $u(x, \lambda)$ in $\Omega$ such that $u(x, \lambda) \rightarrow 0$ as $|x| \rightarrow \infty$ for all $\lambda \geq \lambda^{*}$.

Proof. With $\phi(x)$ as in H11, consider the linear boundary value problem

$$
\begin{cases}-\Delta v+m(x) v=\lambda \phi(x), & x \in \Omega, \\ v(x)=0, & x \in \partial \Omega .\end{cases}
$$

In view of H11, a theorem of Meyers and Serrin [17, Theorem 10, p. 527] shows, if $n \geq 3$, that (5.8) has a nontrivial solution $v=v(x, \lambda)$ in $\Omega$ such that $v(x, \lambda) \rightarrow 0$ as $|x| \rightarrow \infty$.

Let $u_{R}=u_{R}(x, \lambda)$ be the solution of (5.1) in the bounded domain $\Omega_{R}$ guaranteed by Theorem 3.1, for $\lambda \geq \lambda^{*}$ :

$$
\begin{cases}-\Delta u_{R}+m(x) u_{R}=\lambda f\left(x, u_{R}(x, \lambda)\right), & x \in \Omega_{R}, \\ u_{R}(x, \lambda)=0, & x \in \partial \Omega_{R} .\end{cases}
$$

For $x \in \Omega_{R},(5.8)$ and (5.9) give

$$
(-\Delta+m(x))\left(v-u_{R}\right)=\lambda\left[\phi(x)-f\left(x, u_{R}(x, \lambda)\right)\right] .
$$

Since $u_{R}(x, \lambda) \leq T$ for all $x \in \bar{\Omega}_{R}$ by the proof of Theorem 4.3, it follows from (5.8)-(5.10) and H11 that

$$
\begin{cases}(-\Delta+m(x))\left(v-u_{R}\right) \geq 0 & \text { in } \Omega_{R} \\ v-u_{R}=0 & \text { on } \partial \Omega \\ v-u_{R} \geq 0 & \text { on } S_{R} .\end{cases}
$$


Then the maximum principle implies that

$$
0<u_{R}(x, \lambda) \leq v(x, \lambda), \quad x \in \Omega_{R}, \lambda \geq \lambda^{*}>0 .
$$

The sequence $\left\{u_{j}(x, \lambda): j=1,2, \ldots\right\}$ constructed in Lemma 4.2 satisfies, in particular,

$$
0 \leq w_{0}(x, \lambda) \leq u_{j}(x, \lambda) \leq v(x, \lambda)
$$

for all $x \in \Omega \cup \partial \Omega, \lambda \geq \lambda^{*}, j=1,2, \ldots$, where $w_{0}(x, \lambda)$ is defined by (4.1). The proof of monotone convergence of the sequence $\left\{u_{j}(x, \lambda)\right\}$ to a solution $u(x, \lambda)$ of $(5.1),(5.2)$ is exactly the same as in Theorem 4.3. It also follows from (D) that $u(x, \lambda) \rightarrow 0$ as $|x| \rightarrow \infty$ since $v(x, \lambda) \rightarrow 0$ and the convergence of $\left\{u_{j}(x, \lambda)\right\}$ to $u(x, \lambda)$ holds pointwise in $\Omega$ for all $\lambda \geq \lambda^{*}$.

For example, the results of this section apply to the equation

$$
\begin{aligned}
& -\Delta u+m(x) u=\lambda p(x)\left(u^{\gamma}-\varepsilon u^{\beta}\right), \\
& \qquad x \in \Omega, 1<\gamma<\beta, \varepsilon>0, \lambda>0 .
\end{aligned}
$$

Hypotheses H3-H5 hold automatically, and the other hypotheses of this section reduce to the following:

$\mathrm{H} 1-\mathrm{H} 2 . m$ and $p$ are nonnegative, $m, p \in C_{\mathrm{loc}}^{\alpha}(\Omega)$, and $p$ is not identically zero.

H8. $m$ is uniformly positive.

H9. $p(x)$ is bounded.

H10. $\lim _{|x| \rightarrow \infty} p(x)=0$.

H11. $\max _{|x|=r} p(x) \leq \delta(r) / r^{2}, r \geq r_{0} \geq 0$, where $\delta(r)$ is a Dini function.

Results parallel to Theorems 5.2 and 5.3 hold in the entire space $R^{n}$ if Hypotheses $H j$ are replaced by formally identical Hypotheses $H^{\prime} j$ with $R^{n}$ replacing $\Omega$ and $\partial \Omega$ deleted.

THeOREM 5.4. Suppose that $\mathrm{H}^{\prime} 1-\mathrm{H}^{\prime} 5, \mathrm{H}^{\prime} 8$, and $\mathrm{H}^{\prime} 10$ are satisfied. Then there exists $\lambda^{*}>0$ such that, for all $\lambda \geq \lambda^{*}$, equation (5.1) has a positive solution $u(x, \lambda)$ in $R^{n}$ satisfying

$$
u(x, \lambda) \leq C(\lambda) \exp \left[-\sqrt{m_{0} / 2}|x|\right], \quad x \in R^{n},
$$

for some constant $C(\lambda)>0$. 
THEOREM 5.5. Suppose that $n \geq 3$, and $\mathrm{H}^{\prime} 1-\mathrm{H}^{\prime} 5$ and $\mathrm{H}^{\prime} 11$ are satisfied. Then there exists $\lambda^{*}>0$ such that, for all $\lambda \geq \lambda^{*}$, equation (5.1) has a positive solution $u(x, \lambda)$ in $R^{n}$ such that $u(x, \lambda) \rightarrow 0$ as $|x| \rightarrow \infty$.

Theorem 5.4 is proved from the solution constructed in Theorem 4.5 by application of Kato's theorem. The method used for Theorem 5.3 also proves Theorem 5.5.

6. Existence theorems in the case of negative $m(x)$. It will now be proved, under modified hypotheses, that the existence theorems of $\$ 4$ remain valid when $m(x)$ has negative values in $\Omega$. Although $m(x)$ will be allowed to change sign for arbitrarily large $|x|$, the set $\left\{x \in R^{n}: m(x)<0\right\}$ will be required to have sufficiently large measure so that the linear operator $L$ in (1.3) is nodally oscillatory in $\Omega$, i.e. for arbitrary $R>0$ there exists a nonempty bounded domain $M \subset G_{R} \cap \Omega$ such that the Dirichlet problem

$$
L u=0 \quad \text { in } M, \quad u=0 \text { on } \partial M
$$

has a nontrivial nonnegative solution $u_{M}$. Many explicit nodal oscillation criteria are known [26]; for example, $L$ is nodally oscillatory in $R^{2}$ if the entries of the matrix $\left(a_{i j}(x)\right)$ are bounded and

$$
\int_{\Omega} m(x) d x=-\infty, \quad n=2 .
$$

Hypotheses $\mathrm{H} 2$ and $\mathrm{H} 5$ will be retained while $\mathrm{H} 1$ and $\mathrm{H} 3$ need to be slightly modified:

$\mathrm{H}^{*} 1$. The $a_{i j}$ are as in $\mathrm{H} 1, m \in C_{\mathrm{loc}}^{\alpha}(\Omega)$, and $L$ is nodally oscillatory in $\Omega$.

$\mathrm{H}^{*} 3$. There exists $T>0$ such that $f(x, T)=0, f(x, t) \geq 0$ if $0 \leq t<T$, and $f(x, t)<0$ if $t>T$ for all $x \in \Omega$.

By $\mathrm{H}^{*} 1$ and the linearity of $L,(6.1)$ has a nontrivial nonnegative solution $u_{M}$ in some nonempty bounded domain $M \subset \Omega$ satisfying $u_{M}(x)$ $\leq T$ for all $x \in M$. Then $\mathrm{H}^{*} 3$ implies that

$$
f\left(x, u_{M}(x)\right) \geq 0 \text { for all } x \in M .
$$

Let $R$ be a positive number such that $M \subset \Omega_{R}$. By H5 there exists a positive constant $K_{j}$, for each $j=1,2, \ldots$, such that both

(i) $m(x)+K_{j}>0$ for all $x \in \Omega_{R+j}$; and

(ii) $\lambda f(x, t)+K_{j} t$ is a nondecreasing function of $t$ in $[0, T]$ for each fixed $x \in \Omega_{R+j}$. 
The lemma below is then easily established by the procedure used for Lemmas 4.1 and 4.2.

LEMMA 6.1. Suppose that $\mathrm{H}^{*} 1, \mathrm{H} 2, \mathrm{H}^{*} 3$, and $\mathrm{H} 5$ are satisfied. Let $u_{M} \in C^{2+\alpha}(\bar{M})$ be a nontrivial nonnegative solution of (6.1) chosen so that $u_{M}(x) \leq T$ for all $x \in M$, and suppose that $M \subset \Omega_{R}$. Then there exists $a$ sequence of functions $u_{j}$ in $\Omega \cup \partial \Omega$ with properties (A), (B), (C), (E) of Lemma 4.2 and

$\left(\mathrm{D}^{*}\right) \quad w_{0}(x) \leq u_{j}(x, \lambda) \leq T$ for all $x \in \Omega \cup \partial \Omega, j=1,2, \ldots, \lambda>0$, where $w_{0}(x)=u_{M}(x)$ if $x \in \bar{M}$ and $w_{0}(x)=0$ otherwise.

TheOREM 6.2. If $\mathrm{H}^{*} 1, \mathrm{H} 2, \mathrm{H}^{*} 3$, and $\mathrm{H} 5$ hold, then the boundary value problem (1.1), (1.2) has a bounded positive solution $u(x, \lambda)$ in $\Omega$ such that $w_{0}(x) \leq u(x, \lambda) \leq T$ for all $x \in \Omega \cup \partial \Omega$ and for all $\lambda>0$, where $w_{0}(x)$ is defined in Lemma 6.1.

THEOREM 6.3. Suppose that the hypotheses of Theorem 6.2 hold in the entire space $R^{n}$ (with $\partial \Omega$ deleted). Then equation (1.1) has a bounded positive solution satisfying $w_{0}(x) \leq u(x, \lambda) \leq T$ for all $x \in R^{n}$ and for all $\lambda>0$.

The proofs on the basis of Lemma 6.1 are essentially the same as the proof of Theorem 4.3.

\section{REFERENCES}

[1] K. Ako, On the Dirichlet problem for quasilinear elliptic differential equations of the second order, J. Math. Soc. Japan, 13 (1961), 45-62.

[2] H. Amann, Existence and multiplicity theorems for semilinear elliptic boundary value problems, Math. Z., 150 (1976), 281-295.

[3] H. Amann and M. G. Crandall, On some existence theorems for semilinear elliptic equations, Indiana Univ. Math. J., 27 (1978), 779-790.

[4] C. Bandle, Existence theorems, qualitative results and a priori bounds for a class of nonlinear Dirichlet problems, Arch. Rat. Mech. Anal., 58 (1975), 219-238.

[5] V. Benci and D. Fortunato, Weighted Sobolev spaces and the nonlinear Dirichlet problem in unbounded domains, Ann. Mat. Pura Appl., Ser. 4, 121 (1979), 319-336.

[6] Some nonlinear elliptic problems with asymptotic conditions, Nonlinear Anal., 3 (1979), 157-174.

[7] H. Berestycki and P. L. Lions, Une méthode locale pour l'existence de solutions positives de problèmes semi-linéaires elliptiques dans $R^{N}$, J. Analyse Math., 38 (1980), 144-187.

[8] H. Berestycki, P. L. Lions, and L. A. Peletier, An ODE approach to the existence of positive solutions for semilinear problems in $R^{N}$, Indiana Univ. Math. J., 30 (1981), $141-157$. 
[9] M. S. Berger, On the existence and structure of stationary states for a nonlinear Klein-Gordon equation, J. Functional Anal., 9 (1972), 249-261.

[10] M. S. Berger and M. Schechter, Embedding theorems and quasilinear elliptic boundary value problems for unbounded domains, Trans. Amer. Math. Soc., 172 (1972), 261-278.

[11] D. K. Bose, On semilinear elliptic boundary value problems in unbounded domains, Proc. Roy. Soc. Edinburgh, Sect. A, 77 (1977), 177-192.

[12] R. Courant and D. Hilbert, Methods of Mathematical Physics II, Wiley-Interscience, New York/London, 1962.

[13] D. E. Edmunds and W. D. Evans, Elliptic and degenerate-elliptic operators in unbounded domains, Ann. Scuola Norm. Sup. Pisa, 27 (1973), 591-640.

[14] D. E. Edmunds and J. R. L. Webb, Quasilinear elliptic problems in unbounded domains, Proc. Roy. Soc. London A, 334 (1973), 397-410.

[15] T. Kato, Growth properties of solutions of the reduced wave equation with a variable coefficient, Comm. Pure Appl. Math., XII (1959), 403-425.

[16] H. B. Keller, Elliptic boundary value problems suggested by nonlinear diffusion processes, Arch. Rat. Mech. Anal., 5 (1969), 363-381.

[17] N. Meyers and J. Serrin, The exterior Dirichlet problem for second order elliptic partial differential equations, J. Math. Mech., 9 (1960), 513-538.

[18] M. Nagumo, On principally linear elliptic differential equations of the second order, Osaka Math. J., 6 (1954), 207-229.

[19] E. S. Noussair, On semilinear elliptic boundary value problems in unbounded domains, J. Differential Equations, 41 (1981), 334-348.

[20] E. S. Noussair and C. A. Swanson, Positive solutions of quasilinear elliptic equations in exterior domains, J. Math. Anal. Appl., 75 (1980), 121-133.

[21] A. Ogata, On bounded positive solutions of nonlinear elliptic boundary value problems in an exterior domain, Funcial. Ekvac., 17 (1974), 207-222.

[22] M. H. Protter and H. F. Weinberger, Maximum Principles in Differential Equations, Prentice-Hall, Englewood Cliffs, N. J., 1967.

[23] P. H. Rabinowitz, Pairs of positive solutions of nonlinear elliptic partial differential equations, Indiana Univ. Math. J., 23 (1973), 173-186.

[24] K. Schmitt, Boundary value problems for quasilinear second order elliptic equations, Nonlinear Anal. Theory Methods Appl., 2 (1978), 263-309.

[25] W. A. Strauss, Existence of solitary waves in higher dimensions, Comm. Math. Phys., 55 (1977), 149-162.

[26] C. A. Swanson, Semilinear second order elliptic oscillation, Canad. Math. Bull., 22 (1979), 139-157.

Received October 21, 1982. Support for second author from NSERC (Canada) is acknowledged with gratitude.

UNIVERSITY OF NEW SOUTH WALES

Kensington, N.S.W., Australia 2033

AND

UNIVERSITY OF BRITISH COLUMBIA

VANCOUVER, Canada V6T 1Y4 


\title{
PACIFIC JOURNAL OF MATHEMATICS \\ EDITORS
}

\author{
DoNALD BABBITT (Managing Editor) \\ University of California \\ Los Angeles, CA 90024 \\ J. DugunduI \\ University of Southern California \\ Los Angeles, CA 90089-1113 \\ R. FINN \\ Stanford University \\ Stanford, CA 94305 \\ HERMANN FlasChKa \\ University of Arizona \\ Tucson, AZ 85721
}

C. C. Moore

University of California

Berkeley, CA 94720

ARTHUR OGUS

University of California

Berkeley, CA 94720

Hugo Rossi

University of Utah

Salt Lake City, UT 84112

H. SAMELSON

Stanford University

Stanford, CA 94305

ASSOCIATE EDITORS
R. ARENS
E. F. BECKENBACH
B. H. NeUMANN
F. WOLF
K. YoSHIDA (1906-1982)

\section{SUPPORTING INSTITUTIONS}

UNIVERSITY OF ARIZONA
UNIVERSITY OF BRITISH COLUMBIA
CALIFORNIA INSTITUTE OF TECHNOLOGY
UNIVERSITY OF CALIFORNIA
MONTANA STATE UNIVERSITY
UNIVERSITY OF NEVADA, RENO
NEW MEXICO STATE UNIVERSITY
OREGON STATE UNIVERSITY
UNIVERSITY OF ARIZONA
CALIFORNIA INSTITUTE OF TECHNOLOGY
UNIVERSITY OF CALIFORNIA
UNIVERSITY OF NEVADA, RENO
OREGON STATE UNIVERSITY
UNIVERSITY OF OREGON
UNIVERSITY OF SOUTHERN CALIFORNIA
STANFORD UNIVERSITY
UNIVERSITY OF HAWAII
UNIVERSITY OF TOKYO
UNIVERSITY OF UTAH
WASHINGTON STATE UNIVERSITY
UNIVERSITY OF WASHINGTON 


\section{Pacific Journal of Mathematics}

Vol. 115, No. $1 \quad$ September, 1984

Carlos Andradas Heranz and José Manuel Gamboa Mutuberría, A note on projections of real algebraic varieties $\ldots \ldots \ldots \ldots \ldots \ldots \ldots \ldots \ldots \ldots$

Jürgen Appell and Maria Patrizia Pera, Noncompactness principles in nonlinear operator approximation theory $\ldots \ldots \ldots \ldots \ldots \ldots \ldots \ldots \ldots \ldots$

Timothy John Carlson, Extending Lebesgue measure by infinitely many

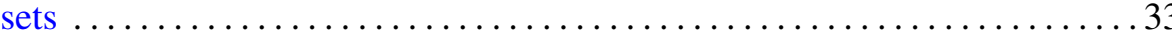

Donald S. Coram and Paul Frazier Duvall, Jr., Non-cell-like

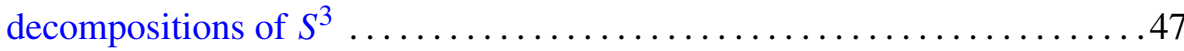

Edward Norman Dancer, Order intervals of selfadjoint linear operators and

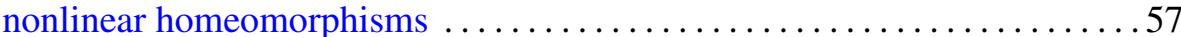

Ralph Jay De Laubenfels, Well-behaved derivations on $C[0,1] \ldots \ldots \ldots 73$

D. Feyel and A. de La Pradelle, Sur certaines extensions du théorème

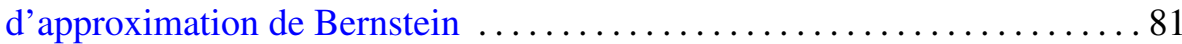

Colin C. Graham and Bertram Manuel Schreiber, Bimeasure algebras on

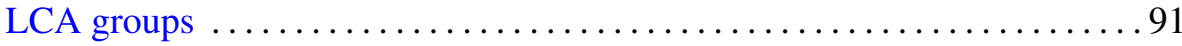

Richard Howard Hudson, Class numbers of imaginary cyclic quartic fields

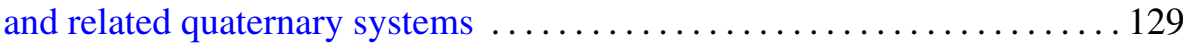

Carl Groos Jockusch, Jr. and Iraj Kalantari, Recursively enumerable sets and van der Waerden's theorem on arithmetic progressions . . . . . . . . 143

J. F. McClendon, On noncontractible valued multifunctions ........... 155 Akihiko Miyachi, Weak factorization of distributions in $H^{p}$ spaces $\ldots \ldots \ldots 165$ Ezzat S. Noussair and Charles Andrew Swanson, Global positive solutions of semilinear elliptic problems

Jon Christopher Snader, Strongly analytic subspaces and strongly

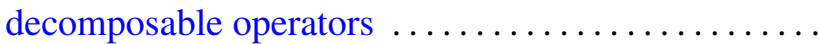

Boguslaw Tomaszewski, A construction of inner maps preserving the Haar measure on spheres

Akihito Uchiyama, The Fefferman-Stein decomposition of smooth functions and its application to $H^{p}\left(\mathbf{R}^{n}\right) \ldots \ldots \ldots$ 